\title{
A Multilingual Semantic Wiki Based on Attempto Controlled English and Grammatical Framework
}

\author{
Kaarel Kaljurand ${ }^{1}$ and Tobias Kuhn ${ }^{1,2}$ \\ 1 Institute of Computational Linguistics, University of Zurich, Switzerland \\ ${ }^{2}$ Chair of Sociology, in particular of Modeling and Simulation, ETH Zurich, Switzerland \\ \{kaljurand, kuhntobias\} @gmail.com
}

\begin{abstract}
We describe a semantic wiki system with an underlying controlled natural language grammar implemented in Grammatical Framework (GF). The grammar restricts the wiki content to a well-defined subset of Attempto Controlled English (ACE), and facilitates a precise bidirectional automatic translation between ACE and language fragments of a number of other natural languages, making the wiki content accessible multilingually. Additionally, our approach allows for automatic translation into the Web Ontology Language (OWL), which enables automatic reasoning over the wiki content. The developed wiki environment thus allows users to build, query and view OWL knowledge bases via a user-friendly multilingual natural language interface. As a further feature, the underlying multilingual grammar is integrated into the wiki and can be collaboratively edited to extend the vocabulary of the wiki or even customize its sentence structures. This work demonstrates the combination of the existing technologies of Attempto Controlled English and Grammatical Framework, and is implemented as an extension of the existing semantic wiki engine AceWiki.
\end{abstract}

Keywords: semantic wiki, multilinguality, controlled natural language, Attempto Controlled English, Grammatical Framework.

\section{Introduction}

Wikis are user-friendly collaborative environments for building knowledge bases in natural language. The most well-known example is Wikipedia, an encyclopedia that is being built by around 100,000 users in hundreds of different languages, with numerous other wikis for smaller domains. Semantic wikis [4] combine the main properties of wikis (ease of use, read-write, collaboration, linking) with knowledge engineering technology (structured content, knowledge models in the form of ontologies, automatic reasoning). Semantic wiki editors simultaneously work with the natural language content and its underlying formal semantics representation. The resulting wikis offer more powerful content management functions, e.g. dynamically created pages based on semantic queries and detection of semantic errors in the content, but have to somehow meet the challenge of keeping the user interface as simple as expected from wikis. The existing semantic wiki engines (e.g. Semantic Mediawik的, Freebase 2 ) support the

${ }^{1}$ http://semantic-mediawiki.org/

2 http://www.freebase.com/

P. Cimiano et al. (Eds.): ESWC 2013, LNCS 7882, pp. 427 441, 2013.

(C) Springer-Verlag Berlin Heidelberg 2013 
inclusion of semantics in the form of RDF-like subject-predicate-object triples, e.g. typed wikilinks (predicates) between two articles (the subject and the object).

Our approach to semantic wikis is based on controlled natural language (CNL) [30]. A CNL is a restricted version of a natural language. For CNLs like Attempto Controlled English (ACE) [9], the syntax is precisely defined, the sentences have a formal (executable) meaning, and they come with end-user documentation describing syntax, semantics and usage patterns. CNLs and their editing tools support the creation of texts that are natural yet semantically precise, and can thus function well in human-machine communication. CNL-based wikis — such as AceWiki [16], on which our approach is based - can offer greater semantic expressivity compared to traditional semantic wikis (e.g. OWL instead of RDF), but their user interface is easier to work with (because it is still based on natural language).

In this paper we describe a semantic wiki system with an underlying controlled natural language grammar implemented in Grammatical Framework (GF). The grammar restricts the wiki editors into a well-defined subset of ACE that is automatically translatable into the Web Ontology Language (OWL) [11] and thus enables automatic semantic reasoning over the wiki content. Additionally, the grammar facilitates a precise bidirectional automatic translation between ACE and language fragments of a number of other natural languages. The developed wiki environment thus allows users to build, query and view OWL knowledge bases via a user-friendly multilingual natural language interface. The underlying multilingual grammar is integrated into the wiki itself and can be collaboratively edited to extend the vocabulary and even customize the multilingual representations of ACE sentences. Our work demonstrates the combination of the existing technologies of $\mathrm{ACE}$ and GF, and is implemented by extending the existing ACE-based semantic wiki engine AceWiki with support to multilinguality and collaborative GF grammar editing. The main goal of this work is to explore natural language grammar based semantic wikis in the multilingual setting. As a subgoal we ported a fragment of ACE to several natural languages (other than English) in a principled way by implementing a shared abstract syntax. The wiki environment allows us to test the usefulness of this work and furthermore collaboratively improve the initial ports. The overall work is part of the the EU research project MOLTd 3 .

This paper is structured as follows: in Section 2 we review related work; in Section 3 we introduce the core features of the existing tools and technologies employed in the rest of the paper (namely ACE, GF and AceWiki); in Section 4 we describe the multilingual GF-implementation of ACE; in Section 5 we discuss the extension of AceWiki based on the GF-implementation of ACE; in Section 6 we provide an initial evaluation of our system; in Section 7 we summarize our main results and outline future work.

\section{Related Work}

The related work falls into several categories such as multilingual CNLs, CNL-based wikis, multilingual wikis, multilingual ontologies, and ontology verbalization.

Many general purpose and domain-specific controlled natural languages have been developed based on many different natural languages [24]. However, there has not been

${ }^{3}$ http: / / www.molto-project.eu 
an effort to bring them under the same semantic model or synchronize their development in a community-driven manner [22]. Our multilingual ACE grammar is an experiment in this direction. A multilingual version of ACE (in GF) was first investigated in [28]. Our current implementation is partly an extension of this work. A similar work is [12], which builds a bidirectional interface between a controlled fragment of Latvian and OWL, using ACE as an interlingua, and implementing the interface using GF.

The main CNL-based wiki that we are aware of is AceWiki which is also the basis of our work and will be discussed below. [10] describes the MoKi semantic wiki engine which offers a "lightly-structured access mode" for its structured content (OWL). In this mode the content is displayed as an uneditable ACE text; editing is supported for the simpler isA and partOf statements using templates that combine CNL with HTMLforms, or using a native OWL syntax. In terms of multilinguality our wiki system has some similarities with the OWL ontology editor described in [2] which allows the user to view the ontology in three CNLs, two based on English and one on Chinese. As the main difference compared to these systems, our system uses the CNLs as the only user interface for both editing and viewing.

The research on GF has not yet focused on a wiki-like tool built on top of a GFbased grammar or application. Tool support exists mostly for users constructing single sentences (not texts) and working alone (not in collaboration). A notable exception is [23], which investigates using GF in a multilingual wiki context, to write restaurant reviews on the abstract language-independent level by constructing GF abstract trees.

Even though the mainstream wiki engines generally allow for the wiki articles to be written in multiple languages, these different language versions exist independently of each other and only article-level granularity is offered by the system for interlinking the multilingual content. Some recent work targets that problem though, e.g. the EU project CoSyne 4 develops a technology for the multilingual content synchronization in wikis by using machine translation.

Ontology languages (such as RDF, OWL and SKOS) typically support languagespecific labels as attachments to ontological entities (such as classes and properties). Although the ontological axioms can thus be presented multilingually, their keywords (e.g. SubClassOf, some, only) are still in English and their syntactic structure is not customizable. This is clearly insufficient for true ontology verbalization, especially for expressive ontology languages like OWL as argued in [6], which describes a sophisticated lexical annotation ontology to be attached to the domain ontology as linguistic knowledge. Our work can also be seen as attaching (multilingual) linguistic knowledge to a semantic web ontology. [7] discusses a multilingual CNL-based verbalization of business rules. It is similar to our approach by being implemented in GF but differs by not using OWL as the ontology language.

\section{Underlying Technologies}

\subsection{Attempto Controlled English}

Attempto Controlled English (ACE) [9] is a general purpose CNL based on first-order logic. ACE can be viewed as both a natural language understandable to every English

\footnotetext{
${ }^{4}$ http: //www. cosyne.eu/
} 
speaker, as well as a formal language with a precisely defined syntax and semantics understandable to automatic theorem proving software. ACE offers many language constructs, the most important of which are countable and mass nouns (e.g. 'man', 'water'); proper names ('John'); generalized quantifiers ('at least 2'); indefinite pronouns ('somebody'); intransitive, transitive and ditransitive verbs ('sleep', 'like', 'give'); negation, conjunction and disjunction of noun phrases, verb phrases, relative clauses and sentences; and anaphoric references to noun phrases through definite noun phrases, pronouns, and variables. Texts built from these units are deterministically interpreted via Discourse Representation Structures (DRS) [15], which can be further mapped to formats supported by existing automatic reasoners (e.g. OWL, SWRL, FOL, TPTP). The ACE sentence structures and their unambiguous interpretations are explained in the end-user documentation in the form of construction and interpretation rules.

The grammar of ACE and its mapping to DRS cannot be modified by the end-users but they can customize ACE in their applications by specifying a content word lexicon of nouns, verbs, adjectives, adverbs and prepositions and their mapping to logical atoms.

While originally designed for software specifications, in the recent years ACE has been developed with the languages and applications of the Semantic Web in mind. [13] describes ACE fragments suitable for mapping to and from languages like OWL, SWRL and DL-Query. ACE View [14] and AceWiki are ACE-based tools for building OWL ontologies. The study described in [20] provides evidence that ACE is a user-friendly language for specifying OWL ontologies, providing a syntax that is easier to understand and use compared to the standard OWL syntaxes.

\subsection{Grammatical Framework}

Grammatical Framework (GF) [27] is a functional programming language for building multilingual grammar applications. Every GF program consists of an abstract syntax (a set of functions and their categories) and a set of one or more concrete syntaxes which describe how the abstract functions and categories are linearized (turned into surface strings) in each respective concrete language. The resulting grammar describes a mapping between concrete language strings and their corresponding abstract trees (structures of function names). This mapping is bidirectional — strings can be parsed to trees, and trees linearized to strings. As an abstract syntax can have multiple corresponding concrete syntaxes, the respective languages can be automatically translated from one to the other by first parsing a string into a tree and then linearizing the obtained tree into a new string.

While GF can be used to build parsers and generators for formal languages, it is optimized to handle natural language features like morphological variation, agreement, and long-distance dependencies. Additionally, the GF infrastructure provides a resource grammar library (RGL), a reusable grammar library of the main syntactic structures and morphological paradigms currently covering about 30 natural languages [26]. As the library is accessible via a language-independent API, building multilingual applications remains simple even if the programmers lack detailed knowledge of the linguistic aspects of the involved languages. These features make GF a good framework for the implementation of CNLs, especially in the multilingual setting [29]. The development of GF has focused on parsing tools, grammar editors, and extending the grammar 
library to new languages. The current algorithm for parsing GF grammars is based on Parallel Multiple Context-Free Grammars and allows for incremental parsing, which enables look-ahead editing [1].

\subsection{AceWiki}

AceWiki [18] is a CNL-based semantic wiki engine, implemented in Java using the Echo Web Framework. It uses ACE as the content language and OWL as its underlying semantic framework integrating its main reasoning tasks (consistency checking, classification and query answering) and making them available via the ACE-based interface.

The content of an AceWiki instance is written in a subset of ACE formally defined in a grammar notation called Codeco [19]. The grammar targets an OWL-compatible fragment of ACE, i.e. ACE sentences that are semantically outside of the OWL expressivity cannot be expressed in the wiki. This guarantees that all of the AceWiki content can be automatically translated to OWL in the background. Additionally, the grammar is used to drive a look-ahead editor which guides the input of a new sentence by proposing only syntactically legal continuations of the sentence.

The AceWiki content is structured into a set of articles, each article containing a sequence of entries which are either declarative sentences (corresponding to OWL axioms) or questions (corresponding to OWL class expressions). Additionally informal comments are supported. Upon every change in the wiki, an OWL reasoner determines its effect and possibly flags inconsistencies or updates the dynamically generated parts of the wiki (e.g. concept hierarchies and answers to questions).

The content words (proper names, nouns, transitive verbs, relational nouns and transitive adjectives) in the wiki sentences map one-to-one (i.e. link) to wiki articles. Semantically, content words correspond to OWL entities: proper names to OWL individuals, nouns to OWL classes, and the relational words to OWL properties.

\section{Multilingual ACE}

In order to provide a multilingual interface to AceWiki, we implemented the syntax of ACE in GF and ported it via the RGL API to multiple natural language fragments. (See the ACE-in-GF website 7 and [5] for more details of this work.) On the ACE side, the grammar implements the subset supported by the AceWiki Codeco grammar and can be thus automatically tested against the Codeco implementation to verify the coverage and precision properties. The implementation accesses the GF English resource grammar through the language-independent API (Figure 1). This API makes it easy to plug in other RGL-supported languages. Our current implementation targets 15 European languages. Most of them provide full coverage of the ACE syntactic structures, for some languages a few structures (e.g. verb phrase coordination, some forms of questions) have not been implemented yet.

\footnotetext{
${ }^{5}$ http://attempto.ifi.uzh.ch/acewiki/

6 http://echo.nextapp.com/

${ }^{7}$ http://github.com/Attempto/ACE-in-GF
} 


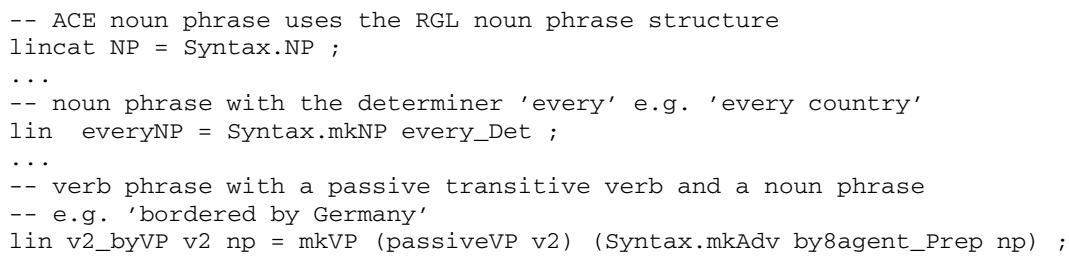

Fig. 1. Fragment of a GF grammar for ACE listing the linearization rules for the functions everyNP and v2_byVP. There are around 100 such rules. This GF module (functor) implements the ACE sentence structures via RGL's API calls (e.g. every_Det, mkVP). A concrete language implementation parametrizes this functor with a concrete language resource (English in case of ACE) and possibly overrides some of the rules with language-specific structures. For the function categories, the grammar uses categories that are also used in the ACE user-level documentation, e.g. noun (N), transitive verb (V2), noun phrase (NP), relative clause.

While most of the multilingual grammar can be written in a language-neutral format, the lexicon modules are language dependent. Different languages have different morphological complexity, e.g. while the Codeco-defined AceWiki subset of ACE operates with two noun forms (singular and plural) and three verb forms (infinitive, 3rd person singular and past participle), other languages (e.g. Finnish) might need many more forms to be used in the various ACE sentence structures. Fortunately, we can make use of the RGL calls, e.g. mkN ("make noun") and mkV2 ("make transitive verb"), to create the necessary (language-specific) lexicon structures from a small number of input arguments (often just the lemma form), using the so called smart paradigms [26].

In order to view an ACE text in another language, one needs to parse it to an abstract tree which can then be linearized into this language (Figure 2). This makes it possible to map various natural language fragments to the formal languages that are supported by ACE (e.g. OWL and TPTP) and verbalize such formal languages via ACE (if this is supported) into various natural language fragments (Figure 3). For example, the OWL-to-ACE verbalizer [13] can be used as a component in a tool that makes an OWL ontology viewable in a natural language, say Finnish. This tool must contain a lexicon, i.e. a mapping of each OWL entity to the Finnish word that corresponds to the ACE category that the verbalizer assigns to the OWL entity.

While the ACE concrete syntax is designed to be unambiguous, i.e. every supported sentence generates just a single abstract tree, the grammar in general does not guarantee this property for the other implemented languages. In some cases it seems to be better to let a user work with an ambiguous representation if it offers a simpler syntax and if the ambiguity can be always explained (e.g. via the ACE representation) or removed in the actual usage scenario (e.g. in a collaborative wiki environment).

\section{AceWiki-GF}

Our multilingual semantic wiki based on ACE and GF has been realized as an extension of AceWiki, and is thus (preliminarily) called AceWiki-GF. Extending AceWiki 


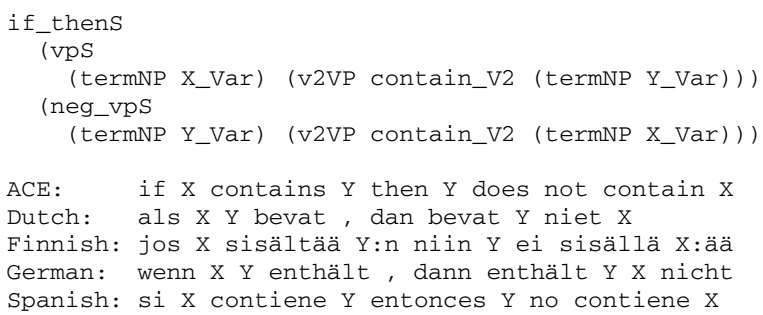

Fig. 2. Abstract tree and its linearizations into five languages which express the OWL asymmetric property axiom, which is assigned by the ACE-to-OWL mapping to the ACE sentence. The linearizations feature different word orders depending on the language. The tree abstracts away from linguistic features like word order, case, and gender, although it still operates with syntactic notions such as negated verb phrase.

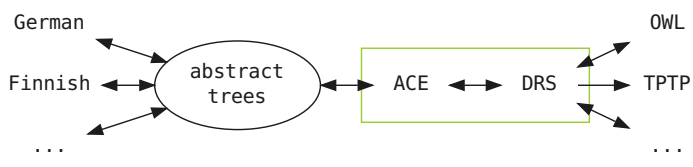

Fig. 3. Bidirectional mapping between a formal language like OWL and a natural language like Finnish facilitated by the multilingual GF-implementation of ACE and various mappings between $\mathrm{ACE}$ and other formal languages.

has allowed us to reuse its infrastructure (such as look-ahead editing, access to OWL reasoners, the presentation of reasoning results, and document navigation). In the following we only describe the main differences and extensions. (See Section 3.3 for the general discussion of the AceWiki engine.)

Because AceWiki is a monolingual engine, several modifications had to be done to accommodate multilinguality, i.e. to support viewing/editing in multiple languages depending on the users' preferences:

- the Codeco grammar/parser for ACE was replaced by the GF-implemented multilingual ACE grammar and a GF parser;

- the English-specific lexicon editor was replaced by a simple GF source editor which can be used to edit any GF grammar modules, among them lexicon modules;

- the atomic wiki entry, which for the monolingual AceWiki was an ACE sentence, was changed to a GF abstract tree set. The new representation is language-neutral and can furthermore represent ambiguity, as explained in Section 4 ,

- the notion of wiki article/page was extended to also include arbitrarily named pages (in AceWiki all pages are named by their corresponding OWL entity) and pages that represent editable grammar modules.

The existing AceWiki user interface has largely been preserved; the main additions are the disambiguation dialog and a menu for setting the content language, which also 


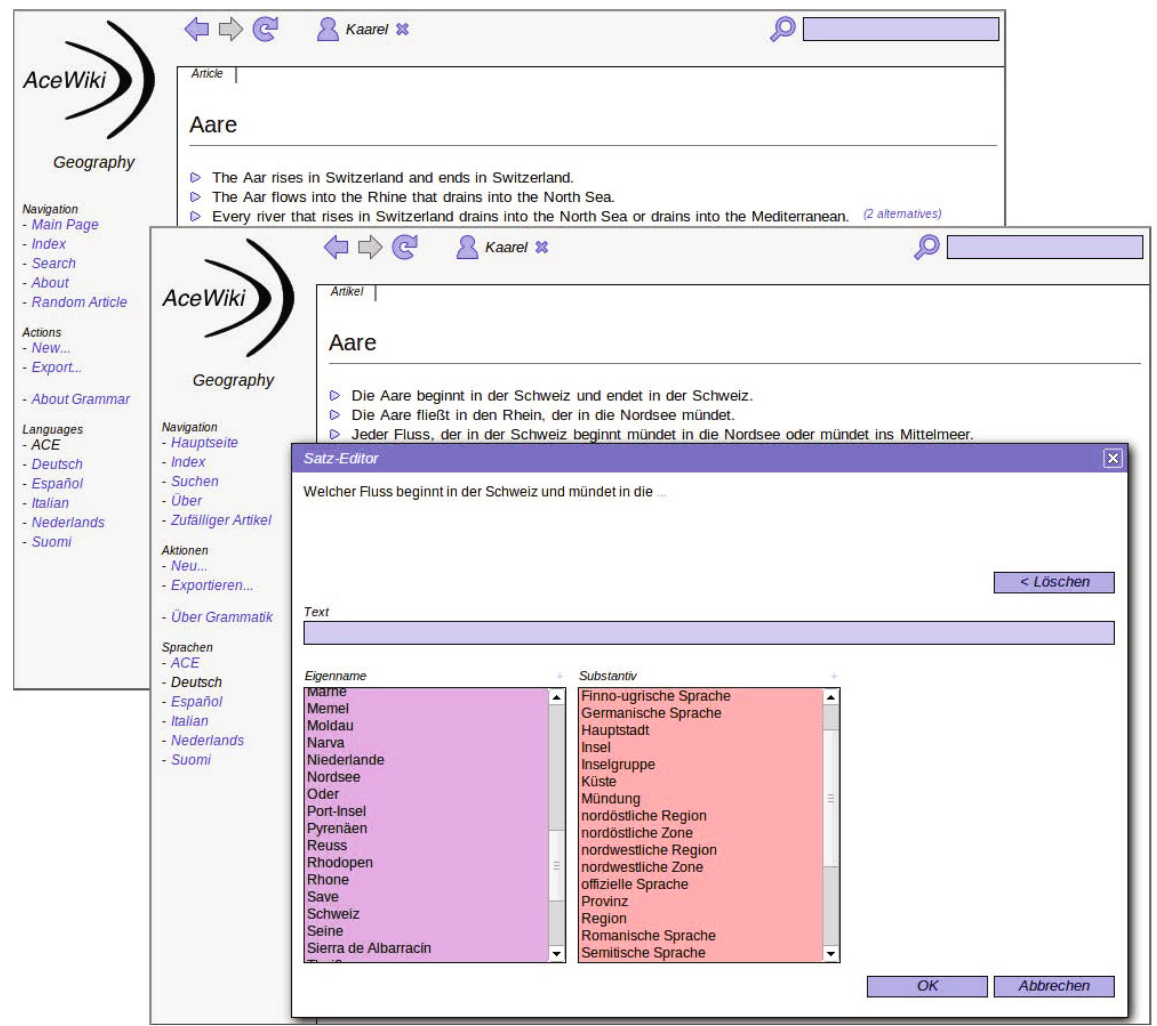

Fig. 4. Multilingual geography article displayed in ACE and German. The wiki language (of both the content and the user interface) can be changed in the left sidebar. Otherwise the user interface is the same as in AceWiki, with the look-ahead editor that helps to input syntactically controlled sentences, in this case offering proper names and common nouns as possible continuations.

determines the user interface language (Figure 4). The wiki still follows the main principle of CNL-based wikis, i.e. that formal notations are hidden. In our case the user does not see the trees which actually define the content but only interacts with the natural language sentences. (Experienced users can still look at the GF parser output providing information on syntax trees, translation alignment diagrams, etc.)

\subsection{Structure and Linking}

In general, AceWiki-GF follows the AceWiki structure - the wiki is a set of articles, each containing a sequence of sentences. New is the fact that also the grammar definition is part of the wiki and can be referenced from the articles using wikilinks.

A GF grammar is structured in a way that is naturally representable as a set of wiki articles. Each grammar module can be stored as a wiki article and linked to the modules that it imports. Furthermore, grammar modules have internal structure - sets of 
categories and functions (which reference categories) — which can be linked to wiki content because the content is represented as a set of trees (i.e. structures of function names). One of the benefits of having a grammar definition as part of the wiki is that it provides an integrated documentation of the language that the wiki users are required to use. Note that the full grammar contains also modules which are part of the general RGL and thus not editable and also not part of the wiki. This resource is made accessible via external links to the online RGL browser 8 .

\subsection{Sentence Editing}

The user interface for adding and modifying wiki entries is the same as in AceWiki, i.e. based on sentences and supporting the completion of a syntactically correct sentence by displaying a list of syntactically legal words that can follow the partially completed sentence. The language of the sentence depends on the chosen wiki language. In case an entry is ambiguous (i.e. parsing results in multiple trees) then the ambiguity is preserved. If viewed in another language, multiple different sentences can then occur as linearizations of the ambiguity. This allows the wiki users who work via the other language to resolve the ambiguity. A monolingual way to deal with ambiguity is to implement for every concrete syntax an additional "disambiguation syntax" [29], that overrides the linearizations of the ambiguous constructs to have an unambiguous, although possibly a more formal-looking notation. This syntax could be used to display the entry in the case of ambiguity.

We note that some syntax-aware editors, e.g. the GF Syntax Editol 19 or the OWL Simplified English editor [25], operate more on the abstract tree level and thus avoid the problem of ambiguous entries. These approaches also simplify smaller edits e.g. replacing a word in the beginning of the sentence. The fact that they abstract away from linguistic details like case and gender might make them preferable for users with only basic knowledge of the underlying language. It is therefore worth exploring these editing approaches also in the AceWiki-GF context.

\subsection{Lexicon and Grammar Editing}

Our wiki makes the grammar available as a set of interlinked grammar modules falling into the following categories:

- ACE resource grammar (about 30 modules which are typically identical to their English resource grammar counterparts, sometimes overriding certain structures);

- ACE application grammar, reflecting the AceWiki subset of ACE (one module);

- instantiation of this grammar for each supported language with additional modules that describe language-specific overriding of some of the functions;

- content word lexicon module(s) for each language.

In order to add a new word to the wiki, a line needs to be added to the lexicon wiki page, i.e. the page that corresponds to the lexicon module (Figure 5). Although editing the

\footnotetext{
${ }^{8}$ http://www.grammaticalframework.org/lib/doc/browse/

9 http://cloud.grammaticalframework.org/syntax-editor/editor.html
} 


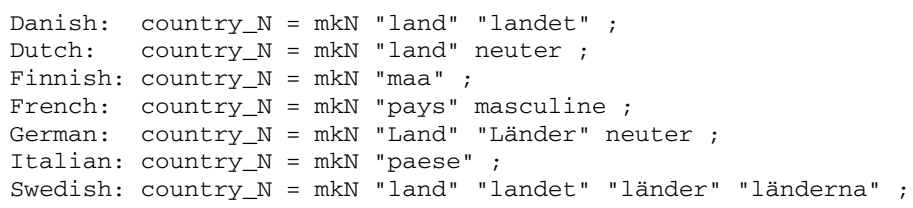

Fig. 5. Entries in the multilingual lexicon. Smart paradigms like mkN are used to create the internal structure of the entry. In many cases giving only the lemma form to the word class operator is sufficient to get a correct internal structure. In some cases further forms or information about gender (in some languages) needs to be added. This makes the user interface to the lexicon relatively simple and homogeneous across languages.

lexicon technically means editing the GF grammar, the lexicon module is conceptually much simpler than the general grammar module and maps one-to-one to the respective ACE lexicon structure (for English). The structure of lexicons in all the supported languages is roughly the same even if some languages are morphologically more complex (e.g. have more case endings). The language-specific lexical structures are hidden from the user behind language-neutral categories like $\mathrm{N}$ and $\mathrm{V} 2$ and constructed by functions like $\mathrm{mkN}$ and $\mathrm{mkV} 2$ which are capable of determining the full word paradigm on the basis of only one or two input forms. Thus, support for multilinguality does not increase the conceptual complexity of the wiki.

Wiki users experienced in GF are also able to modify the full grammar, although we do not see many compelling use cases for that as ACE itself is pre-defined and thus changing its grammar should not be allowed (e.g. it would break the functioning of the mapping to OWL). Its verbalization to other languages, however, is sometimes a matter of taste, and could be therefore made changeable by the wiki users, e.g. users can add an alternative formulation of an ACE sentence in some language by using a GF variant. Also, the possibility to define arbitrary GF operators can make certain lexicon entry tasks more convenient.

A change to the underlying grammar (even if only in the lexicon module) can have the following consequences for the content: (1) removing a function can render some of the wiki entries (the ones whose trees use this function) invalid, the user must then reformulate the respective sentences to conform to the new grammar/lexicon; (2) altering the linearization of a function might cause some sentences to become unparsable or ambiguous in the corresponding language. This does not have an immediate effect on the stored wiki content because the storage is based on trees, but if an existing sentence is submitted again to the parser then it might fail or result in more trees than before. A general change to a grammar module (e.g. removing a category) can also make the whole grammar invalid, which obviously should be avoided.

\subsection{Underlying Semantic Representation}

As in the original AceWiki, each AceWiki-GF entry has a corresponding OWL representation. It is obtained by linearizing the abstract tree of the entry as an ACE sentence (using the multilingual grammar) and then translating it to OWL (as defined in [13]). 
In the ACE representation each content word is annotated with its word class information and the corresponding OWL entity, which is currently derived from the lemma form of the ACE word. Ambiguous wiki entries map in general to multiple OWL forms (although this is not necessarily the case). Such entries are not included in the underlying semantic representation.

\subsection{Multilinguality}

The content of our wiki can be currently made available in up to 15 languages, which form a subset of the RGL that has been tested in the context of the multilingual ACE grammar. In principle every RGL language (that exists now or will be added to the RGL in the future) can be plugged in, because we access the RGL via its languageneutral API. However, language-specific customization of some of the phrase structures is usually necessary as discussed in Section 4.

For a concrete wiki instance a smaller number of languages might be initially preferred and more translations of the wiki content could be added gradually. In addition to the wiki reader and the wiki editor, there is now a third type of a wiki user, namely the translator. Their main task is to translate all existing words by referencing the correct operators in the RGL morphological API and to check if the automatically generated translations are accurate with respect to ACE. The skillset for this task therefore includes the knowledge of ACE and the RGL morphology API.

\subsection{Implementation}

Apart from having been implemented as an extension of AceWiki, the discussed wiki engine is supported by two external (and independently developed) tools. First, the GF Webservice [3] provides linearization and parsing (and the related look-ahead) services for GF grammars. The GF Webservice has been recently extended to provide a GF Cloud Service AP10 which additionally allows for modifications to the grammar. Secondly, the ACE parser APE 11 provides the mapping of ACE sentences to the OWL form (as is the case also for the monolingual AceWiki). The current implementation of AceWiki-GF is available on GitHub 12 and can be used via some demo wikis 13 .

\section{Evaluation}

In previous work, two usability experiments have been performed on AceWiki with altogether 26 participants [17]. The results showed that AceWiki and its editor component are easy to learn and use. Another study confirmed that writing ACE sentences with the editor is easier and faster than writing other formal languages [21]. It has also been demonstrated that ACE is more effective than the OWL Manchester Syntax in terms

\footnotetext{
${ }^{10}$ http://cloud.grammaticalframework.org/gf-cloud-api.html

11 http://github.com/Attempto/APE

12 http://github.com/AceWiki/AceWiki

13 http: / / attempto.ifi.uzh.ch/acewiki-gf/
} 
of understandability [20]. As these previous studies did not include the multilinguality features, the evaluations presented below focus on the multilingual grammar aspects.

We first evaluated how many syntactically correct sentences of the AceWiki ACE subset the multilingual grammar accepts. To that aim, we used the AceWiki Codeco testset which is an exhaustive set of sentences with length of up to 10 tokens $(19,422$ sentences, disregarding some deprecated ACE sentences) [18]. The GF-based ACE grammar successfully covers all these sentences.

Next, we measured the syntactic precision by randomly generating large numbers of sentences at different tree depths and parsing them with both the ACE parser and the Codeco parser. The precision of the grammar was found to be sufficient although not perfect. The main deficiency compared to the Codeco grammar is the lack of DRS-style anaphoric reference modeling. In practice this means that some accepted sentences will be rejected by the ACE-to-OWL translator on the grounds of containing unresolvable definite noun phrases. Ignoring such sentences the precision was $98 \%$ (measured at tree depth of 4 for which the sentence length is 11 tokens on average).

The ambiguity level of ACE sentences (of the Codeco testset) was found to be $3 \%$. In these relatively rare cases, involving complex sentences, the grammar assigns two abstract trees to an input ACE sentence. This is always semantically harmless ambiguity (i.e. it would not manifest itself in translations) resulting from the rules for common nouns and noun phrases which accept similar input structures. While the coverage and precision are measures applicable only to the ACE grammar (because an external definition and a reference implementation of ACE exists), the ambiguity can be measured for all the languages implemented in the grammar by linearizing trees in a given language and checking if the result produces additional trees when parsed. Some semantically severe ambiguities were found using this method (e.g. occasional subject/object relative clause ambiguity in Dutch and German triggered by certain combinations of case and gender, double negation ambiguity in some Romance languages). These findings can either be treated in the grammar i.e. in the design of the respective controlled languages or highlighted in the wiki environment in a way that they can be effectively dealt with.

To measure the translation quality we looked at the translations of $40 \mathrm{ACE}$ sentences using 20 lexicon entries. The sentences were verbalizations of a wide variety of OWL axiom structures (also used in [20]). We wanted to check whether the meaning in all the languages adheres to the precise meaning of OWL statements. The translations covered nine languages (Catalan, Dutch, Finnish, French, German, Italian, Spanish, Swedish, and Urdu) and were checked by native speakers to evaluate the translations with respect to the original ACE sentence and the ACE interpretation rules [5]. In general, the translations were found to be acceptable and accurate although several types of errors were found, mainly caused by the fact that the lexicon creators were not very familiar with the respective languages. Concretely, the following four error types were observed:

RGL Errors. Some problems (e.g. missing articles in Urdu) were traced to errors in the resource grammar library, and not in our ACE application grammar.

Incorrect Use of Smart Paradigms. Several mistakes were caused by an incorrect use of the RGL smart paradigms, either by applying a regular paradigm to an irregular word or simply providing the operator with an incorrect input (e.g. a genitive form instead of a nominative). 
Stylistic Issues. A further problem were stylistic issues, i.e. structures that are understandable but sound unnatural to a native speaker, e.g. using an inanimate pronoun to refer to a person.

Negative Determiners. We experienced that translating sentences with negative determiners such as "no', e.g. "every man does not love no woman" or "no man does not love a woman" can result in meaning shifts between languages. This was eventually handled by extending the RGL to include noun phrase polarity.

Most of these problems are easy to fix by a native speaker with GF skills. (We assume that if there is enough interest in the port of a particular wiki into another language, it should be possible to find such a person.) A more conclusive evaluation is planned that includes the wiki environment and uses a larger real-world vocabulary.

\section{Discussion and Future Work}

The main contribution of our work is the study of CNL-based knowledge engineering in a semantic wiki environment. The main novelty with respect to previous work is making the wiki environment multilingual. As the underlying technologies we have used Attempto Controlled English, which is a syntactically user-friendly formal language and provides a mapping to the expressive ontology language OWL, and Grammatical Framework, which was used to provide a multilingual bidirectional interface to ACE covering several natural languages. We have built the implementation on top of AceWiki, an existing monolingual semantic wiki engine. In order to make our system multilingual, the architecture of AceWiki was generalized. Although the underlying implementation has become more complex, the user interface has largely remained the same. On the (multilingual) lexicon editing side, this is mainly due to the support for smart paradigms that GF provides via its RGL. In the future, we plan to use the grammar-based approach also to implement the other aspects of the wiki, such as multilingual user interface labels (see [23]).

The current approach generates the OWL representations using the existing ACEto-OWL translator. An alternative method is to implement this translator also in GF. In this way the users would have full control over what kind of OWL axioms can be generated because they can edit the OWL mapping (concrete syntax) in the wiki. The semantic aspects of the wiki could also be generalized to allow for any kind of ACEbased reasoning, offered by tools like RACE [8] or TPTP reasoners.

The presented work can be also extended in various more general directions. Although the current system is ACE-based, its general architecture allows for any grammar to be used as the basis of the wiki content as long as it is implemented in GF. Such alternative grammars might not map naturally to a language like OWL and are thus less interesting in the context of the Semantic Web. Examples are grammars for a tourist phrase book, a museum catalog, a technical manual, or a collection of mathematics exercises. Such wikis would mainly profit from the supported multilinguality and not so much from semantic web style reasoning, or may need other forms of reasoning.

Another direction is to improve the grammar editing features of the environment and to develop the system into a tool for collaboratively designing CNLs. The wiki users could take e.g. the ACE grammar as starting point and customize it for a specific 
domain, possibly changing some of its original features and design decisions. The wiki sentences could then serve as unit/regression test sets to check the currently effective grammar implementation.

Acknowledgments. The research leading to these results has received funding from the European Union's Seventh Framework Programme (FP7/2007-2013) under grant agreement FP7-ICT-247914. The authors would like to thank Norbert E. Fuchs for useful comments on the draft of this paper.

\section{References}

1. Angelov, K.: The Mechanics of the Grammatical Framework. PhD thesis, Chalmers University of Technology (2011)

2. Bao, J., Smart, P.R., Shadbolt, N., Braines, D., Jones, G.: A Controlled Natural Language Interface for Semantic Media Wiki. In: 3rd Annual Conference of the International Technology Alliance, ACITA 2009 (September 2009)

3. Bringert, B., Angelov, K., Ranta, A.: Grammatical Framework Web Service. In: Proceedings of EACL 2009 (2009)

4. Bry, F., Schaffert, S., Vrandečić, D., Weiand, K.: Semantic wikis: Approaches, applications, and perspectives. Reasoning Web. Semantic Technologies for Advanced Query Answering, 329-369 (2012)

5. Camilleri, J.J., Fuchs, N.E., Kaljurand, K.: Deliverable D11.1. ACE Grammar Library. Technical report, MOLTO project (June 2012), http: / /www. molto-project.eu/ biblio/deliverable/ace-grammar-library

6. Cimiano, P., Buitelaar, P., McCrae, J., Sintek, M.: Lexinfo: A declarative model for the lexicon-ontology interface. Web Semantics: Science, Services and Agents on the World Wide Web 9(1), 29-51 (2011)

7. Davis, B., Enache, R., van Grondelle, J., Pretorius, L.: Multilingual Verbalisation of Modular Ontologies Using GF and lemon. In: Kuhn, T., Fuchs, N.E. (eds.) CNL 2012. LNCS, vol. 7427, pp. 167-184. Springer, Heidelberg (2012)

8. Fuchs, N.E.: First-Order Reasoning for Attempto Controlled English. In: Rosner, M., Fuchs, N.E. (eds.) CNL 2010. LNCS, vol. 7175, pp. 73-94. Springer, Heidelberg (2012)

9. Fuchs, N.E., Kaljurand, K., Kuhn, T.: Attempto Controlled English for Knowledge Representation. In: Baroglio, C., Bonatti, P.A., Małuszyński, J., Marchiori, M., Polleres, A., Schaffert, S. (eds.) Reasoning Web 2005. LNCS, vol. 5224, pp. 104-124. Springer, Heidelberg (2008)

10. Ghidini, C., Rospocher, M., Serafini, L.: Modeling in a Wiki with MoKi: Reference Architecture, Implementation, and Usages. International Journal on Advances in Life Sciences 4 (2012)

11. W3C OWL Working Group. OWL 2 Web Ontology Language Document Overview (Second Edition). W3C Recommendation. Technical report, W3C (December 11, 2012), http: / /www.w3 .org/TR/owl2-overview/

12. Grūzītis, N.: Formal Grammar and Semantics of Controlled Latvian Language. PhD thesis, University of Latvia (2011)

13. Kaljurand, K.: Attempto Controlled English as a Semantic Web Language. PhD thesis, Faculty of Mathematics and Computer Science, University of Tartu (2007)

14. Kaljurand, K.: ACE View - an ontology and rule editor based on Attempto Controlled English. In: 5th OWL Experiences and Directions Workshop (OWLED 2008), Karlsruhe, Germany, October 26-27, 12 pages (2008) 
15. Kamp, H., Reyle, U.: From Discourse to Logic. Introduction to Modeltheoretic Semantics of Natural Language, Formal Logic and Discourse Representation Theory. Kluwer Academic Publishers, Dordrecht (1993)

16. Kuhn, T.: AceWiki: A Natural and Expressive Semantic Wiki. In: Semantic Web User Interaction at CHI 2008: Exploring HCI Challenges (2008)

17. Kuhn, T.: How Controlled English can Improve Semantic Wikis. In: Lange, C., Schaffert, S., Skaf-Molli, H., Völkel, M. (eds.) Proceedings of the Fourth Workshop on Semantic Wikis, European Semantic Web Conference 2009. CEUR Workshop Proceedings, vol. 464. CEURWS (June 2009)

18. Kuhn, T.: Controlled English for Knowledge Representation. PhD thesis, Faculty of Economics, Business Administration and Information Technology of the University of Zurich (2010)

19. Kuhn, T.: A principled approach to grammars for controlled natural languages and predictive editors. Journal of Logic, Language and Information 22(1), 33-70 (2013)

20. Kuhn, T.: The understandability of OWL statements in controlled English. Semantic Web 4(1), 101-115 (2013)

21. Kuhn, T., Höfler, S.: Coral: Corpus access in controlled language. Corpora 7(2), 187-206 (2012)

22. Luts, M., Tikkerbär, D., Saarmann, M., Kutateladze, M.: Towards a Community-Driven Controlled Natural Languages Evolution. In: Rosner, M., Fuchs, N.E. (eds.) Pre-Proceedings of the Second Workshop on Controlled Natural Languages, CNL 2010. CEUR Workshop Proceedings, vol. 622, CEUR-WS (2010)

23. Meza-Moreno, M.S., Bringert, B.: Interactive Multilingual Web Applications with Grammatical Framework. In: Nordström, B., Ranta, A. (eds.) GoTAL 2008. LNCS (LNAI), vol. 5221, pp. 336-347. Springer, Heidelberg (2008)

24. Pool, J.: Can Controlled Languages Scale to the Web? In: 5th International Workshop on Controlled Language Applications (2006)

25. Power, R.: OWL Simplified English: A Finite-State Language for Ontology Editing. In: Kuhn, T., Fuchs, N.E. (eds.) CNL 2012. LNCS, vol. 7427, pp. 44-60. Springer, Heidelberg (2012)

26. Ranta, A.: The GF Resource Grammar Library. Linguistic Issues in Language Technology 2(2) (2009)

27. Ranta, A.: Grammatical Framework: Programming with Multilingual Grammars. CSLI Publications, Stanford (2011), ISBN-10: 1-57586-626-9 (Paper), 1-57586-627-7 (Cloth)

28. Angelov, K., Ranta, A.: Implementing Controlled Languages in GF. In: Fuchs, N.E. (ed.) CNL 2009. LNCS, vol. 5972, pp. 82-101. Springer, Heidelberg (2010)

29. Ranta, A., Enache, R., Détrez, G.: Controlled Language for Everyday Use: The MOLTO Phrasebook. In: Rosner, M., Fuchs, N.E. (eds.) CNL 2010. LNCS, vol. 7175, pp. 115-136. Springer, Heidelberg (2012)

30. Wyner, A., Angelov, K., Barzdins, G., Damljanovic, D., Davis, B., Fuchs, N., Hoefler, S., Jones, K., Kaljurand, K., Kuhn, T., Luts, M., Pool, J., Rosner, M., Schwitter, R., Sowa, J.: On controlled natural languages: Properties and prospects. In: Fuchs, N.E. (ed.) CNL 2009. LNCS, vol. 5972, pp. 281-289. Springer, Heidelberg (2010) 